CONDITION THAT THE LINE COMMON TO $N-1$

PLANES IN AN $N$ SPACE MAY PIERCE A GIVEN QUADRIC SURFACE IN THE

SAME SPACE.

BY DR. VIRGIL SNYDER.

(Read at the Detroit meeting of the American Association for the Advancement of Science, August 10, 1897.)

THIs note is a generalization of a proof given in a recent paper * of the geometric significance of the sign of a certain determinant. This determinant was the combinant of four linear spherical complexes; the spheres common to the four complexes are real when the combinant is negative.

When applied to linear line complexes, which can be derived from the spherical by an imaginary transformation, I subsequently found, by another method, $\dagger$ that the corresponding determinant is positive when the lines which cut four given ones are real.

The law is general, and will apply to determinants of odd order, and to imaginary transformations.

(1) Let

$$
\sum_{i=1}^{n+1} \alpha_{i, k} x_{i}=0 \quad[k=1,2, \ldots, n \ldots 1]
$$

represent $n-1$ linear equations, homogeneous in $n+1$ variables $x_{i}$; these can be regarded as the equations of $n-1$ planes in space of $n$ dimensions.

Let the variables $x_{i}$ satisfy the homogeneous quadratic equation

$$
\varphi\left(x_{1}, x_{2}, x_{3}, x_{4}, x_{5}, x_{6}\right)=\varphi(x)=0
$$

which may be regarded as the equation of a quadric surface in the same space. The $n-1$ planes will intersect in a line; I propose to give the criterion for the reality of the two points in which this line pierces the given surface. It depends upon the sign of a determinant which may be defined as follows:

Let

$$
y_{1} \frac{\partial \varphi}{\partial x_{1}}+y_{2} \frac{\partial \varphi}{\partial x_{2}}+\cdots+y_{n+1} \frac{\partial \varphi}{\partial x_{n+1}}=0
$$

* "Criteria for nodes in dupin's cyclides," Ann. of Math., vol. 11, No. 5, p. $137 \mathrm{ff}$.

$\dagger$ Bulletin Amer. Math. Soc., vol. 3, No. 7, p. $247 \mathrm{ff}$. 
be the equation of a plane ; make it identical with

$$
a_{1} y_{1}+a_{2} y_{2}+\cdots+a_{n+1} y_{n+1}=0 ;
$$

then

$$
a_{1}=\frac{\partial \varphi}{\partial x_{1}}, a_{2}=\frac{\partial \varphi}{\partial x_{2}}, \cdots a_{n+1}=\frac{\partial \varphi}{\partial x_{n+1}} .
$$

From (5),

$$
a_{1} x_{1}=x_{1} \frac{\partial \varphi}{\partial x_{1}}, \cdots a_{n+1} x_{n+1}=x_{n+1} \frac{\partial \varphi}{\partial x_{n+1}},
$$

hence

$$
\sum_{i=1}^{n+1} a_{i} x_{i}=\sum_{i=1}^{n+1} x_{i} \frac{\partial \varphi}{\partial x_{i}}=2 \varphi(x) .
$$

Differentiating (6),

$$
\sum_{i=1}^{n+1}\left(x_{i} d a_{i}+a_{i} d x_{i}\right)=2 d \varphi .
$$

Again, from (5), $a_{i} d x_{i}=\frac{\partial \varphi}{\partial x_{i}} d x_{i}$, hence

$$
\sum_{i=1}^{n+1} \frac{\partial \varphi}{\partial x_{i}} d x_{i}=\sum_{i=1}^{n+1} a_{i} d x_{i}=d \varphi
$$

then, from (7),

$$
\sum_{i=1}^{n+1} x_{i} d a_{i}=d \varphi
$$

From (5), $x_{i}$ is a linear function of $a_{1} \cdots a_{n+1}$ and $d \varphi$ is a complete differential, hence

$$
d \varphi=\sum_{i=1}^{n+1} \frac{\partial \Phi}{\partial a_{i}} d a_{i}
$$

where $\Phi$ is a homogeneous quadratic function of $\alpha_{i}$ such that

$$
\begin{gathered}
\varphi(x)=\Phi(\alpha) \text { and } x_{i}=\frac{\partial \Phi}{\partial \alpha_{i}} \text { when } \\
a_{i}=\frac{\partial \varphi}{\partial x_{i}} .
\end{gathered}
$$


The combinant of the surface $\varphi(x)=0$ and the $n-1$ planes (1) is of the form

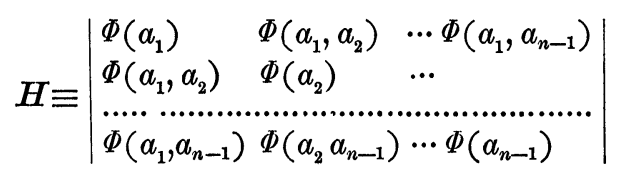

where $\Phi\left(a_{i}\right)$ is $\Phi$ function of the coefficients of the $i^{\text {th }}$ plane

(1): i. e., $k=i$, and $\Phi\left(a_{l}, a_{m}\right)=\sum_{i=1}^{n+1} a_{i, l} \frac{\partial \Phi\left(a_{m}\right)}{\partial a_{i, m}}=\Phi\left(a_{m}, a_{l}\right)$.

The problem of finding $\Phi$ when $\varphi$ is given in its general form is coextensive with reducing $\varphi(x)$ to the form

$$
\sum_{i=1}^{n+1} x_{i}^{2}=0 \text {. }
$$

As every quadric can be reduced to this form, the proof will be restricted to it. (Negative terms in (10) will be considered later.) Solve the system (1) for any $n-1$ of the variables, as $x_{1}, \cdots x_{n-1}$ in terms of the other two, $x_{n}, x_{n+1}$. The general term will be

$$
x_{i}=\frac{\left(\begin{array}{c}
i \\
n
\end{array}\right) x_{n}+\left(\begin{array}{c}
i \\
n+1
\end{array}\right) x_{n+1}}{D}
$$

where $D$ is the determinant $\left(a_{1}, \cdots a_{n-1, n-1}\right)$ and $\left(\begin{array}{c}i \\ n\end{array}\right)$ is what this determinant becomes when the $i^{\text {th }}$ column $a_{i, k}$ has been replaced by $a_{n, k}[k=1,2, \cdots n-1]$.

Substitute these values for $x_{i}$ from (11) in (10); this gives

$$
\begin{gathered}
x_{n}{ }^{2}\left[\sum_{i=1}^{n+1}\left(\begin{array}{c}
i \\
n
\end{array}\right)^{2}+D^{2}\right]+2 x_{n} x_{n+1} \sum_{i=1}^{n-1}\left(\begin{array}{l}
i \\
n
\end{array}\right)\left(\begin{array}{c}
i \\
n+1
\end{array}\right) \\
+x_{n+1}^{2}\left[\sum_{i=1}^{n+1}\left(\begin{array}{c}
i \\
n+1
\end{array}\right)^{2}+D^{2}\right]=0 .
\end{gathered}
$$

The roots of this equation are real and distinct, real and coincident, or imaginary, according as

$$
\Delta \equiv\left[\sum_{i=1}^{n-1}\left(\begin{array}{l}
i \\
n
\end{array}\right)\left(\begin{array}{c}
i \\
n+1
\end{array}\right)\right]^{2}-
$$




$$
\left[\sum_{i=1}^{n-1}\left(\begin{array}{l}
i \\
n
\end{array}\right)^{2}+D^{2}\right]\left[\sum_{i=1}^{n-1}\left(\begin{array}{c}
i \\
n+1
\end{array}\right)^{2}+D^{2}\right]
$$

is positive, zero, or negative.

This expression can be re-arranged into the form

$$
\begin{gathered}
\Delta \equiv-D^{2}\left[\sum_{i=1}^{n-1}\left(\begin{array}{l}
i \\
n
\end{array}\right)^{2}+\sum_{i=1}^{n-1}\left(\begin{array}{c}
i \\
n+1
\end{array}\right)^{2}+D^{2}\right] \\
-\sum_{k=1}^{n-1} \sum_{i=1}^{n-1}\left[\left(\begin{array}{c}
i \\
n
\end{array}\right)\left(\begin{array}{c}
k \\
n+1
\end{array}\right)-\left(\begin{array}{c}
i \\
n+1
\end{array}\right)\left(\begin{array}{l}
k \\
n
\end{array}\right)\right]^{2} .
\end{gathered}
$$

The elements of $H$ are now

$$
\Phi\left(a_{k}\right)=\sum_{i=1}^{n+1} a_{i, k}^{2}, \Phi\left(a_{k}, a_{l}\right)=\sum_{i=1}^{n+1} a_{i, k} \cdot a_{i, l} ;
$$

$H$ can be expressed as the product of the two rectangular arrays

$$
\left\{\begin{array}{ccc}
a_{1,1}, & \cdots & a_{n+1,1} \\
a_{1,2}, & \cdots & a_{n+1,2} \\
\cdots \cdots \cdots \cdots . . . \cdots \cdots \cdots \cdots \cdots \\
a_{1, n+1}, & \cdots & a_{n+1, n+1}
\end{array}\right\} \cdot\left\{\begin{array}{ccc}
a_{1,1}, & \cdots & a_{n+1,1} \\
a_{1,2}, & \cdots & a_{n+1,2} \\
\cdots \cdots \cdots \cdots \cdots & \cdots \cdots \cdots \cdots \cdots \\
a_{1, n+1} & \cdots & a_{n+1, n+1}
\end{array}\right\}
$$

which, when developed, gives

$$
H=\left[\sum_{i=1}^{n-1}\left(\begin{array}{l}
i \\
n
\end{array}\right)^{2}+\sum_{n=1}^{n-1}\left(\begin{array}{c}
i \\
n+1
\end{array}\right)^{2}+D^{2}\right]
$$

+ terms of the form $T_{i}$, where

$$
\sum_{i=1}^{n-1} \sum_{k=1}^{n-1}\left[\left(\begin{array}{l}
i \\
n
\end{array}\right)\left(\begin{array}{c}
k \\
n+1
\end{array}\right)-\left(\begin{array}{l}
k \\
n
\end{array}\right)\left(\begin{array}{c}
i \\
n+1
\end{array}\right)\right]^{2}=D^{2} \sum T_{i} *
$$

Then

$$
\triangle=-D^{2} H \text {. }
$$

The points are real, coincident or imaginary, according as $H$ is negative, zero or positive. So much for the case when all the terms in (10) are positive, but it is not always possible to reduce (2) to the form (10) by a real transformation. A real transformation can always reduce it to the sum and difference of squares, where the excess of terms of one kind over those of the other is a constant, then a pure imaginary transformation to the form (10).

* Cf. Muir's Determinants, p. 309 ff. 
Suppose that any one term, as $x_{\mathrm{e}}{ }^{2}$ has a negative sign in (10); the corresponding terms in $H$ are also negative. Now, by making the transformation $x_{\nu}=x_{\nu}{ }_{[\nu \neq c}=1, \cdots n+1$, $x_{e}=i x_{\epsilon}{ }^{\prime}$, the form is reduced to (10); the modulus of this transformation is $i$, hence to preserve the reality of the common elements $H$ should be multiplied by -1 for every negative term that appears in (10). Further, it makes no difference whether $(10)$ has $m$ positive and $n+1-m$ negative terms, or conversely. If $n+1$ is even, both of these numbers are odd or even together; if $n+1$ is odd, they would be different, but $H$ is now of odd order, and the sign of every term would also be changed. The two changes of sign would be neutralized, hence:

The line common to $n-1$ planes in space of $n$ dimensions pierces a given quadric surface in the same space in points which are real, coincident or imaginary, according as $(-1)^{h+1} \cdot H$ is positive, zero or negative, where $h$ is the number of negative terms which appear in the equation of the quadric when it is reduced to the algebraic sum of squares, and $H$ is the combinant of the surface and the planes.

Applications. $n=2$.

Let $\varphi(x)=x_{2}{ }^{2}-4 x_{1} x_{3}=0$; find the points where the curve cuts $a_{1} x_{1}+a_{2} x_{2}+a_{3} x_{3}=0$.

$$
\begin{gathered}
2 x_{2} x_{2}^{\prime}-4 x_{1} x_{3}^{\prime}-3 x_{3} a_{1}^{\prime}=0 . \\
a_{1}=-4 x_{3}^{\prime} ; a_{2}=2 x_{2}^{\prime} ; a_{3}=-4 x_{1} . \\
\therefore \Phi(a)=\frac{a_{2}{ }^{2}}{4}-\frac{a_{1} \alpha_{3}}{4} .
\end{gathered}
$$

$h=1$, hence the points where the line $a_{1} x_{1}+a_{2} x_{2}+a_{3} x_{3}+0$ cuts the parabola are real when $a_{2}^{2} \geqq a_{1} a_{3}$.

In line coördinates, $n=2$ would express whether a given point lies within or without a given conic.

$n=3$. Two planes and a quadric surface; this case is self dual.

Points common to two circles (in tetracyclical coördinates).

$n=4$. Points common to three spheres (pentaspherical coördinates).

Circles cutting three given circles $c_{1}, c_{2}, c_{3}$ at given angles $\varphi_{1}, \varphi_{2}, \varphi_{3}$.

$n=5$. Lines cutting four given lines, or belonging to 4 linear complexes. 
Spheres cutting 4 given spheres $s_{1}, s_{2}, s_{3}, s_{4}$ at given angles $\varphi_{1}, \varphi_{2}, \varphi_{3}, \varphi_{4}$.

$H$ contains the determinant formed by the discriminant of $\varphi$, bordered with the coefficients of the planes as a factor. This expression is of the same degree as $H$, both in $\alpha_{i, k}$ and the coefficients of $\varphi$, hence the remaining factor is numerical. By comparing corresponding terms in the two expressions, this is seen to be \pm 1 . The sign to be prefixed depends upon $h$ in both cases, but the $H$ method has two advantages, viz.:

$H$ is of order $n-1$; the bordered discriminant is of order $2 n$.

$H$ appears in many other connections; the bordered discriminant would have to be calculated for this purpose.

CoRNELL UNIVERSTTY, July 19, 1897.

\section{FLUID MOTION.}

Hydrodynamics. By Horace Lamb, F. R. S. Cambridge University Press. 1895. 8vo., pp. 604.

The appearance of a new treatise on any branch of higher mathematics rarely calls for anything else than congratulations to the author, and the volume before us is no exception to the rule. The problems of hydrodynamics present so many difficulties and the opportunities for students to obtain a connected view of them are so rare that any additional help is valuable. Professor Lamb, however, has gone much further than merely producing a continuous account of the subject as it stands at the present time. He has given us a treatise which will easily rank first amongst those in the English and perhaps in any language. The only other English treatise of the same scope, that by Basset published in 1888, although an advance on those which had previously appeared, rather suffers by comparison, both in its plan and the manner in which it is carried out.

In looking over the list of authors which Professor Lamb gives in an index, we are struck by the frequency with which four or five names occur, and a closer examination of the references attached to other names reveals the fact that the mathematical development of hydrodynamics has been almost entirely due to these four or five writers. It must be concluded from this, either that some cause has prevented all but a very few mathematicians from seriously 